\title{
Alternative subtraction method in QCD using Nagy-Soper scheme
}

\author{
Michael Kubocz* \\ E-mail: kubocz@physik.rwth-aachen.de
}

\section{Giuseppe Bevilacqua}

E-mail: bevilacqua@physik.rwth-aachen.de

\section{Michał Czakon}

E-mail: mczakon@physik.rwth-aachen.de

\section{Michael Krämer}

Institut für Theoretische Teilchenphysik und Kosmologie, RWTH Aachen University, DâĂŞ52056 Aachen, Germany

E-mail: mkraemer@physik.rwth-aachen.de

\section{Małgorzata Worek}

Fachbereich C, Bergische Universität Wuppertal, D-42097 Wuppertal, Germany

E-mail: worek@physik.uni-wuppertal.de

We briefly review our recent progress in the implementation of the new NLO subtraction formalism based on Nagy-Soper parton showers within the framework of HELAC and show first results.

10th International Symposium on Radiative Corrections (Applications of Quantum Field Theory to Phenomenology) - Radcor2011

September 26-30, 2011

Mamallapuram, India

\footnotetext{
${ }^{*}$ Speaker.
} 


\section{NLO calculations and subtraction schemes}

The analysis of experimental signatures at the LHC require theoretical predictions for differential distributions and cross sections. Since corrections to LHC processes are in general large, higher-order contributions are necessary to match experimental accuracies and reduce the theoretical uncertainty. Furthermore, they decrease renormalization and factorization scale dependence of the cross section. The general structure of any NLO calculation consists of two parts, the virtual corrections and real radiation. In this context, the virtual exchange and real emission of partons lead to divergences. After UV-renormalization, the virtual and real emission cross sections each contain infrared and collinear singularities, which are regularized, usually, using dimensional regularization. In order to allow Monte Carlo simulations, different approaches have been devised for the treatment of the divergent parts originating from different phase space contributions, namely phase space slicing and subtraction. In our case, we follow the subtraction approach, which has proven its superiority in many analyses. Generally, subtraction schemes introduce local counterterms $\mathrm{d} \sigma^{A}$, which match the behavior of the real-emission matrix element $\mathscr{M}_{m+1}$ in each soft and collinear region. Subtracting these counter-terms from real-emission matrix elements and adding back the corresponding one-particle integrated counterparts to the virtual contribution $\mathrm{d} \sigma^{V}$ results in finite integrands for both the virtual correction $\left(\{p\}_{m}\right.$ phase space) and the real contribution $\left(\{p\}_{m+1}\right.$ phase space):

$$
\begin{aligned}
\sigma^{\mathrm{NLO}} & =\int_{m} \mathrm{~d} \sigma^{V}+\int_{m+1} \mathrm{~d} \sigma^{A}+\int_{m+1}\left[\mathrm{~d} \sigma^{R}-\mathrm{d} \sigma^{A}\right] \\
& \rightarrow \int_{m}[\mathrm{~d} \sigma^{V}+\underbrace{\sum_{i} \mathscr{V}_{i} \otimes \mathrm{d} \sigma^{B}}_{\text {integrated }}]+\int_{m+1} \underbrace{\left[\mathrm{d} \sigma^{R}-\sum_{i} \mathscr{D}_{i} \otimes \mathrm{d} \sigma^{B}\right]}_{\text {subtracted real }} .
\end{aligned}
$$

The form of the subtraction terms in the singular limits is dictated by the well known factorization properties of QCD amplitudes

$$
\mathscr{M}_{m+1}\left(\{p\}_{m+1}\right) \longrightarrow \sum_{l} v_{l}\left(\{p\}_{m+1}\right) \otimes \mathscr{M}_{m}\left(\{p\}_{m}\right)
$$

Here $v_{l}$ denotes the splitting function involved in the scattering process and $\mathscr{M}_{m}$ the corresponding Born matrix element. Additionally, a proper mapping between the $\{p\}_{m+1}$ and $\{p\}_{m}$ phase spaces is required, which satisfies momentum conservation and on-shellness.

\section{Setup and implementation}

Several different subtraction schemes exist at NLO, which differ in the phase space mapping relating real-emission and leading-order kinematics. In our approach, we use a new subtraction scheme proposed by Nagy and Soper (NS) in the context of a parton shower with quantum interference $[1,2,3]$. In this scheme, for a given number $(N)$ of particles in the final state, the number of mappings (relating real-emission and leading-order kinematics), as well as the number of subtraction terms (local counter terms) scale with $N^{2}$. This is already an improvement over the other well-established scheme, namely the Catani-Seymour (CS) subtraction scheme [4], in which the 
scaling is with $N^{3}$. Even a constant scaling behavior could be achieved for an $N$-gluon final state within the Madgraph framework [5]. Moreover, the application of the shower splitting functions as subtraction terms in the NS-scheme facilitates the combination of shower and NLO calculations. The small number of counterterms is obtained by treating all final state partons, which do not participate in the splitting as a collective spectator. Here, the spectator momenta are reshuffled via a unique Lorentz-transformation

$$
\Lambda^{\mu}{ }_{v}(\hat{K}, K)=g^{\mu}{ }_{v}-\frac{2(\hat{K}+K)^{\mu}(\hat{K}+K)_{v}}{(\hat{K}+K)^{2}}+\frac{2 \hat{K}^{\mu} K_{v}}{K^{2}},
$$

where $\hat{K}=Q-\left(\hat{p}_{i}+\hat{p}_{j}\right)$ and $K=Q-p_{i}$ denote the momentum of the collective spectator in the $\{p\}_{m+1}$ and $\{p\}_{m}$ parton phase space. Throughout our subtraction scheme all splitting functions are derived from usual QCD vertices, spinors and polarization vectors for on-shell partons. In the case of virtual contributions, a fully analytical integration of the splitting functions over the unresolved parton (splitting variable) has to be performed. Hence, for the final state splitting, the full singularity structure can be extracted in form of the $I$-operator

$$
\int_{m+1} \mathrm{~d} \sigma^{A}=\sum_{i} \int_{m} \mathrm{~d} \sigma^{B} \otimes \int_{1} \mathrm{~d} \mathscr{V}_{i}=\int_{m}\left[\mathrm{~d} \sigma^{B} \otimes I\right] .
$$

In this connection, the splitting variable is parameterized by an azimuthal, a collinear and a soft variable. We follow here a semi-numerical approach, where the azimuthal variable is integrated analytically and collinear and soft variables are integrated numerically via Monte Carlo. The briefly introduced alternative subtraction method is implemented for both massless and massive cases into the HELAC-DIPOLES framework [6], which provides additionally helicity/color description, calculation of color correlated amplitudes and the integration setup in a fully automated way.

\section{Results}

To check our implementation, we performed several numerical and analytical tests partly with already existing analytic results [7]. As an example of the first numerical results of our implementation, we show in Fig. [1-3] comparisons of some basic differential distributions evaluated with the Catani-Seymour and the new Nagy-Soper subtraction scheme for the process $e^{+} e^{-} \rightarrow u \bar{u} g+X$. The comparison was done with $\sqrt{s}=1 \mathrm{TeV}$ using the $k T$-algorithm, and the scale for both factorization and renormalization was set at $1 \mathrm{TeV}$. The cross sections are given in femto-barns and correspond to inclusive cuts $\left(p_{T}(j)>20 \mathrm{GeV}, \Delta R_{j j}>0.8\right.$ and $\left.|y(j)|>2.5\right)$. Notice that the details of the setup are not particularly important for the comparison. The plots demonstrate a strong scheme dependence of the subtracted-real and the I-operator contributions taken separately. Independence on the subtraction scheme is obviously achieved, when all contributions to the real cross section are summed up. We have checked the scheme-independence of all our results within a permille accuracy.

\section{References}

[1] Z. Nagy and D. E. Soper, JHEP 0709, 114 (2007) [arXiv:0706.0017 [hep-ph]]. 
[2] Z. Nagy and D. E. Soper, JHEP 0807, 025 (2008) [arXiv:0805.0216 [hep-ph]].

[3] Z. Nagy and D. E. Soper, JHEP 0803, 030 (2008) [arXiv:0801.1917 [hep-ph]].

[4] S. Catani and M. H. Seymour, Nucl. Phys. B 485, 291 (1997) [Erratum-ibid. B 510, 503 (1998)] [hep-ph/9605323].

[5] R. Frederix, S. Frixione, F. Maltoni and T. Stelzer, JHEP 0910, 003 (2009) [arXiv:0908.4272 [hep-ph]].

[6] M. Czakon, C. G. Papadopoulos and M. Worek, JHEP 0908, 085 (2009) [arXiv:0905.0883 [hep-ph]].

[7] C. H. Chung, M. Kramer and T. Robens, JHEP 1106, 144 (2011) [arXiv:1012.4948 [hep-ph]]. 

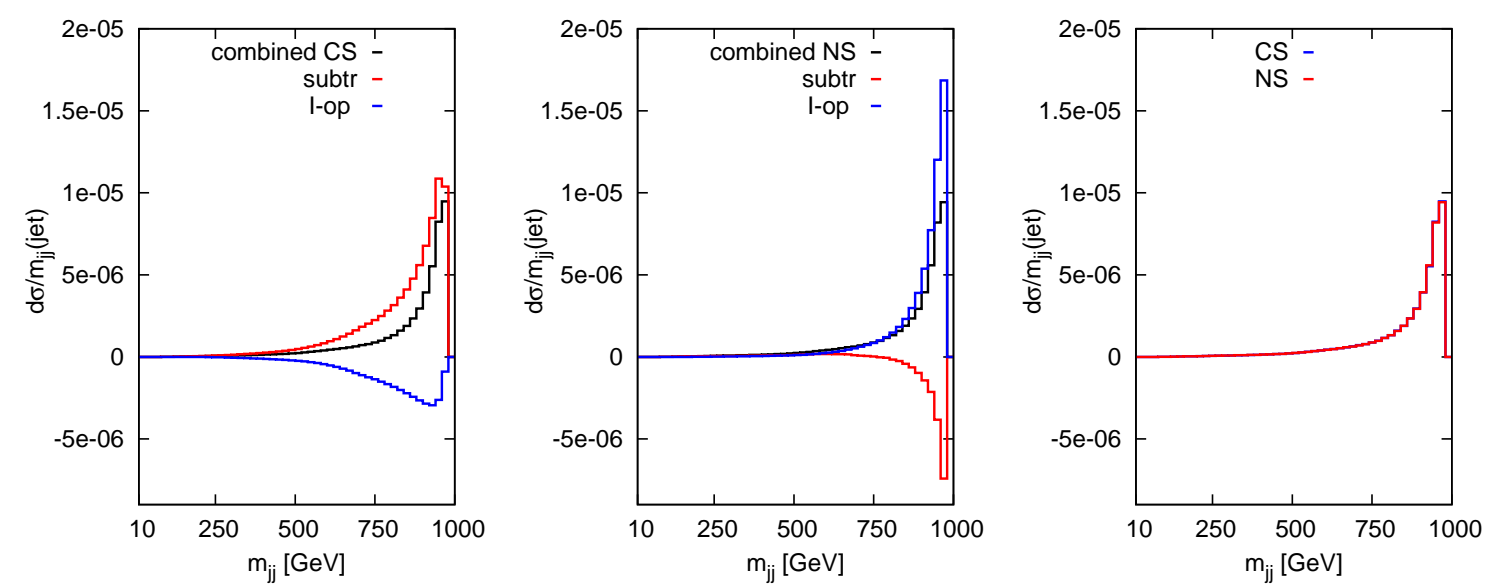

Figure 1: CS vs NS schemes for invariant mass of the two hardest jets.
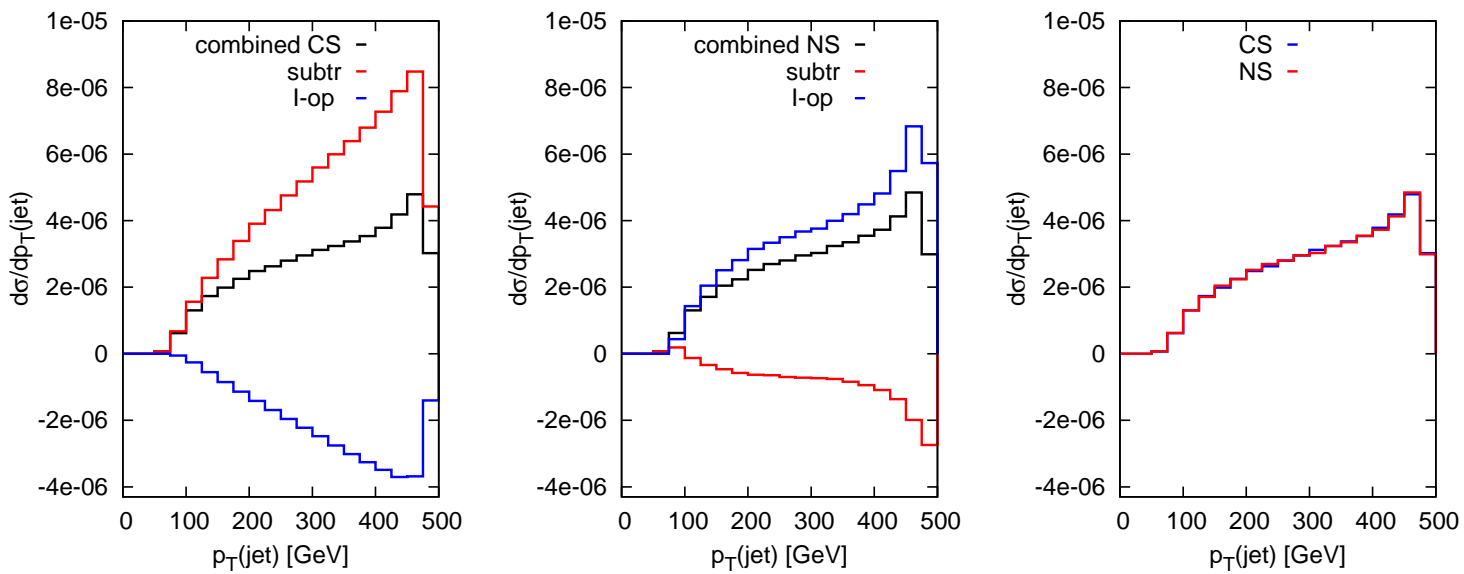

Figure 2: CS vs NS schemes for $p_{T}$ of the hardest jet.
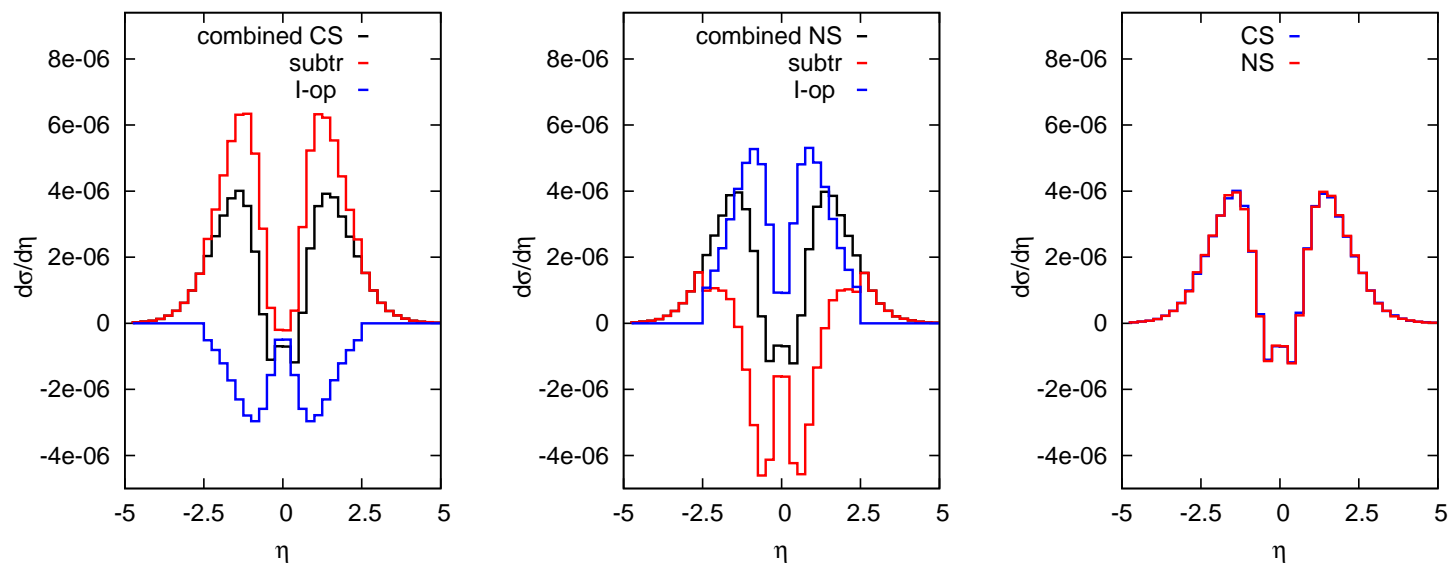

Figure 3: CS vs NS schemes for rapidity of the most forward/backward jet 